PROCEEDINGS OF THE

AMERICAN MATHEMATICAL SOCIETY

Volume 132, Number 11, Pages 3137-3140

S 0002-9939(04)07536-7

Article electronically published on June 21, 2004

\title{
CLASS NUMBERS OF QUADRATIC FIELDS $\mathbb{Q}(\sqrt{D})$ AND $\mathbb{Q}(\sqrt{t D})$
}

\author{
DONGHO BYEON
}

(Communicated by David E. Rohrlich)

\begin{abstract}
Let $t$ be a square free integer. We shall show that there exist infinitely many positive fundamental discriminants $D>0$ with a positive density such that the class numbers of quadratic fields $\mathbb{Q}(\sqrt{D})$ and $\mathbb{Q}(\sqrt{t D})$ are both not divisible by 3 .
\end{abstract}

\section{INTRODUCTION}

Let $r$ and $s$ be the 3-rank of the ideal class group of a real quadratic field $\mathbb{Q}(\sqrt{D})$ and an imaginary quadratic field $\mathbb{Q}(\sqrt{-3 D})$. Scholz $[7$ showed that

$$
r \leq s \leq r+1 .
$$

This is a classical case of Leopoldt's reflection theorem. On the other hand, the Davenport-Heilbronn theorem 3 and a subsequent refinement by Nakagawa and Horie [6] state that there exist infinitely many positive fundamental discriminants $D>0$ with a positive density such that the class numbers of the imaginary quadratic fields $\mathbb{Q}(\sqrt{-3 D})$ are not divisible by 3. Thus we can make the following observation:

There exist infinitely many positive fundamental discriminants $D>$

0 with a positive density such that the class numbers of quadratic

fields $\mathbb{Q}(\sqrt{D})$ and $\mathbb{Q}(\sqrt{-3 D})$ are both not divisible by 3 .

Recently, combining this observation and the Gross-Zagier theorem [1, 2] on the Heegner points and derivatives of $L$-series, Vatsal [8] obtained a positive proportion of rank-one quadratic twists of the modular elliptic curve $X_{0}(19)$. The aim of this paper is to extend the above observation to the pair of fields $\mathbb{Q}(\sqrt{D})$ and $\mathbb{Q}(\sqrt{t D})$ with any square free integer $t$.

Theorem 1.1. Let $t$ be a square free integer. Then there exist infinitely many positive fundamental discriminants $D>0$ with a positive density such that the class numbers of quadratic fields $\mathbb{Q}(\sqrt{D})$ and $\mathbb{Q}(\sqrt{t D})$ are both not divisible by 3 .

From this theorem and the class number product formula of bicyclic biquadratic fields, due to Kubota [5, we can easily obtain the following corollary.

Received by the editors December 23, 2002.

2000 Mathematics Subject Classification. Primary 11R11, 11R29.

This work was supported by grant No. R08-2003-000-10243-0 from the Basic Research Program of the Korea Science \& Engineering Foundation.

(C)2004 American Mathematical Society 
Corollary 1.2. Let $t$ be a square free integer such that the class number of the quadratic field $\mathbb{Q}(\sqrt{t})$ is not divisible by 3 . Then there exist infinitely many bicyclic biquadratic fields $\mathbb{Q}(\sqrt{t}, \sqrt{D})$ whose class number is not divisible by 3 .

Finally, as an application, we shall use Theorem 1.1 to get another positive proportion of rank-one twists of the modular elliptic curve $X_{0}(19)$.

Remark. For the complementary question, Komatsu 4] explicitly constructed a family of infinite pairs of quadratic fields $\mathbb{Q}(\sqrt{D})$ and $\mathbb{Q}(\sqrt{t D})$ whose class numbers are both divisible by 3 , for any square free integer $t$.

\section{Preliminaries}

We recall the result of Nakagawa and Horie in [6], which is a refinement of the result of Davenport and Heilbronn in 3 . Let $m$ and $N$ be two positive integers satisfying the following condition:

(*) If an odd prime number $p$ is a common divisor of $m$ and $N$, then $p^{2}$ divides $N$ but not $m$. Further if $N$ is even, then (i) 4 divides $N$ and $m \equiv 1(\bmod 4)$, or (ii) 16 divides $N$ and $m \equiv 8$ or $12(\bmod$ 16).

For any positive real number $X>0$, we denote by $S_{+}(X)$ the set of positive fundamental discriminants $D<X$ and by $S_{-}(X)$ the set of negative fundamental discriminants $D>-X$, and put

$$
\begin{aligned}
& S_{+}(X, m, N):=\left\{D \in S_{+}(X) \mid D \equiv m(\bmod N)\right\}, \\
& S_{-}(X, m, N):=\left\{D \in S_{-}(X) \mid D \equiv m(\bmod N)\right\} .
\end{aligned}
$$

Theorem 2.1 (Nakagawa and Horie). Let $D$ be a fundamental discriminant and $r_{3}(D)$ the 3 -rank of the quadratic field $\mathbb{Q}(\sqrt{D})$. Then for any two positive integers $m, N$ satisfying $(*)$,

$$
\lim _{X \rightarrow \infty} \sum_{D \in S_{+}(X, m, N)} 3^{r_{3}(D)} / \sum_{D \in S_{+}(X, m, N)} 1=\frac{4}{3}
$$

and

$$
\lim _{X \rightarrow \infty} \sum_{D \in S_{-}(X, m, N)} 3^{r_{3}(D)} / \sum_{D \in S_{-}(X, m, N)} 1=2 .
$$

From Theorem 2.1 and the fact that

$$
\begin{aligned}
& \sum_{\substack{D \in S_{ \pm}(X, m, N) \\
r_{3}(D)=0}} 3^{r_{3}(D)}+3\left(\sum_{D \in S_{ \pm}(X, m, N)} 1-\sum_{\substack{D \in S_{ \pm}(X, m, N) \\
r_{3}(D)=0}} 3^{r_{3}(D)}\right) \\
\leq & \sum_{D \in S_{ \pm}(X, m, N)} 3^{r_{3}(D)},
\end{aligned}
$$

we can easily obtain the following lemma.

Lemma 2.2. Let $D$ be a fundamental discriminant and $h(D)$ the class number of the quadratic field $\mathbb{Q}(\sqrt{D})$. Then for any two positive integers $m, N$ satisfying $(*)$,

$$
\liminf _{X \rightarrow \infty} \frac{\sharp\left\{D \in S_{+}(X, m, N) \mid h(D) \not \equiv 0(\bmod 3)\right\}}{\sharp S_{+}(X, m, N)} \geq \frac{5}{6}
$$


and

$$
\liminf _{X \rightarrow \infty} \frac{\sharp\left\{D \in S_{-}(X, m, N) \mid h(D) \not \equiv 0(\bmod 3)\right\}}{\sharp S_{-}(X, m, N)} \geq \frac{1}{2} .
$$

\section{Proof of theorem 1.1}

Theorem 1.1 follows from the following proposition.

Proposition 3.1. Let $t$ be a square free integer and let $m, N$ be two positive integers satisfying $(*)$ and $(m, t)=1$. Then there exist infinitely many positive fundamental discriminants $D \equiv m(\bmod N)$ with a positive density such that the class numbers of quadratic fields $\mathbb{Q}(\sqrt{D})$ and $\mathbb{Q}(\sqrt{t D})$ are both not divisible by 3 .

Proof. We shall give the details of the case $t \equiv 1(\bmod 16)$ and $(m N, t)=1$, because the other cases are routine modifications of this case. Let

$$
S_{+}^{\prime}(X, m, t N):=\left\{t D \mid D \in S_{+}(X, m, t N)\right\} .
$$

Since $t$ is relatively prime to any $D \in S_{+}(X, m, t N)$, we have $\sharp S_{+}^{\prime}(X, m, t N)=$ $\sharp S_{+}(X, m, t N)$ and

$$
\begin{aligned}
& S_{+}^{\prime}(X, m, t N)=S_{+}\left(t X, t m, t^{2} N\right) \quad \text { if } t \text { is positive, } \\
& S_{+}^{\prime}(X, m, t N)=S_{-}\left(-t X, t m, t^{2} N\right) \text { if } t \text { is negative. }
\end{aligned}
$$

Note that two positive integers $m, t N$ satisfy the condition $(*)$. Then from Lemma 2.2, we have

$$
\liminf _{X \rightarrow \infty} \frac{\sharp\left\{D \in S_{+}(X, m, t N) \mid h(D) \not \equiv 0(\bmod 3)\right\}}{\sharp S_{+}(X, m, t N)} \geq \frac{5}{6} .
$$

Assume $t$ is positive. Since $t m, t^{2} N$ also satisfy $(*)$, we know that

$$
\begin{aligned}
& \liminf _{X \rightarrow \infty} \frac{\sharp\left\{D \in S_{+}^{\prime}(X, m, t N) \mid h(D) \not \equiv 0(\bmod 3)\right\}}{\sharp S_{+}^{\prime}(X, m, t N)} \\
= & \liminf _{X \rightarrow \infty} \frac{\sharp\left\{D \in S_{+}\left(X, t m, t^{2} N\right) \mid h(D) \not \equiv 0(\bmod 3)\right\}}{\sharp S_{+}\left(X, t m, t^{2} N\right)} \geq \frac{5}{6} .
\end{aligned}
$$

Suppose that

(4) $\liminf _{X \rightarrow \infty} \frac{\sharp\left\{D \in S_{+}(X, m, t N) \mid h(D) \not \equiv 0 \text { and } h(t D) \not \equiv 0(\bmod 3)\right\}}{\sharp S_{+}(X, m, t N)}<\frac{2}{3}$.

Then (4) contradicts (3) and we get

$$
\liminf _{X \rightarrow \infty} \frac{\sharp\left\{D \in S_{+}(X, m, t N) \mid h(D) \not \equiv 0 \text { and } h(t D) \not \equiv 0(\bmod 3)\right\}}{\sharp S_{+}(X, m, t N)} \geq \frac{2}{3} .
$$

If we assume that $n$ is negative, then we have

$$
\liminf _{X \rightarrow \infty} \frac{\sharp\left\{D \in S_{+}^{\prime}(X, m, t N) \mid h(D) \not \equiv 0(\bmod 3)\right\}}{\sharp S_{+}^{\prime}(X, m, t N)} \geq \frac{1}{2} .
$$

By a similar argument in the case $t$ is positive, we can obtain

$$
\liminf _{X \rightarrow \infty} \frac{\sharp\left\{D \in S_{+}(X, m, t N) \mid h(D) \not \equiv 0 \text { and } h(t D) \not \equiv 0(\bmod 3)\right\}}{\sharp S_{+}(X, m, t N)} \geq \frac{1}{3} .
$$

The proof is completed since $S_{+}(X, m, t N)$ has a positive density in $S_{+}(X, m, N)$. 


\section{Application to RANK-ONE TWists}

We shall follow Vatsal's paper [8]. Let $t<0$ be a negative square free integer such that 19 splits in the imaginary quadratic field $K=\mathbb{Q}(\sqrt{t})$ and $t \equiv 1(\bmod 4)$. Let $c>0$ be a positive square free integer satisfying the following conditions:

(i) $c \equiv 1(\bmod 4)$ and $(c, t \cdot 19)=1$,

(ii) 19 is inert in $K=\mathbb{Q}(\sqrt{c})$,

(iii) the class numbers of quadratic fields $\mathbb{Q}(\sqrt{c})$ and $\mathbb{Q}(\sqrt{t \cdot c})$ are both not divisible by 3 .

Let $E$ be the modular elliptic curve $X_{0}(19)$ and $L(s, E)$ the corresponding $L$-series. We denote $\psi$ and $\psi^{\prime}$ the quadratic Galois characters associated to $k=\mathbb{Q}(\sqrt{c})$ and $k^{\prime}=\mathbb{Q}(\sqrt{t \cdot c})$. Then the Gross-Zagier theorem [1], 2] on the Heegner points and derivatives of $L$-series implies that if $c$ satisfies the above conditions (i)-(iii), then $L(s, E \otimes \psi)$ has a simple zero and $L\left(s, E \otimes \psi^{\prime}\right)$ is non-zero at $s=1$.

On the other hand, we can find two positive integers $m, N$, which depend on $\{4,19, t\}$ and satisfy $(*)$, such that if $c$ satisfies the congruence $c \equiv m(\bmod N)$, then $c$ satisfies conditions (i) and (ii). From Proposition 3.1, we know that a positive proportion of $c \equiv m(\bmod N)$ satisfies condition (iii). Finally, if we add the condition $3 \mid c$, which is different from Vatsal's $(3, c)=1$, then we have another positive proportion of rank-one twists of $X_{0}(19)$.

\section{REFERENCES}

[1] B. Gross, Heegner points on $X_{0}(N)$, Modular forms (R. Rankin, ed.), Chichester, Ellis Horwood Company, 1984. MR 86f:11003

[2] B. Gross and D. Zagier, Heegner points and derivatives of $L$-series, Invent. Math. 84 (1986), 225-320. MR 87j:11057

[3] H. Davenport and H. Heilbronn, On the density of discriminants of cubic fields II, Proc. Roy. Soc. London A, 322 (1971), 405-420. MR 58:10816

[4] T. Komatsu, An infinite family of pairs of quadratic fields $\mathbb{Q}(\sqrt{D})$ and $\mathbb{Q}(\sqrt{m D})$ whose class numbers are both divisible by 3, Acta Arith., 104 (2002), 129-136. MR 2003f:11166

[5] T. Kubota, Über die Beziehung der Klassenzahlen der Unterkörper des Bizyklischen Biquadratichen Zahlkörpers, Nagoya Math. J. 6 (1953), 119-127. MR 15:605e

[6] J. Nakagawa and K. Horie, Elliptic curves with no torsion points, Proc. A.M.S. 104 (1988), 20-25. MR 89k:11113

[7] A. Scholz, Über die Beziehung der Klassenzahlen quadratischer Körper zueinander, J. Reine Angew. Math., 166 (1932), 201-203.

[8] V. Vatsal, Rank-one twists of a certain elliptic curve, Mathematische Annalen, 311 (1998), 791-794. MR 99i:11050

School of Mathematical Sciences, Seoul National University, Seoul 151-747, Korea

E-mail address: dhbyeonmath.snu.ac.kr 